\title{
NUMERICAL STUDY OF UNSTEADY SUPERCAVITATION PERTURBED BY A PRESSURE WAVE
}

\author{
J. G. ZHENG \\ Temasek Laboratories, National University of Singapore \\ Singapore 117411 Singapore \\ tslzhen@nus.edu.sg \\ B. C. $\mathrm{KHOO}$ \\ Temasek Laboratories, National University of Singapore \\ Singapore 117411 Singapore \\ mpekbc@nus.edu.sg
}

Published 22 June 2016

\begin{abstract}
The unsteady features of supercavitation disturbed by an introduced pressure wave are investigated numerically using a one-fluid cavitation model. The supercavitating flow is assumed to be the homogeneous mixture of liquid and vapour which are locally under both kinetic and thermodynamic equilibrium. The compressibility effects of liquid water are taken into account to model the propagation of pressure wave through flow and its interaction with supercavitation bubble. The interaction between supercavity enveloping an underwater flat-nose cylinder and pressure wave is simulated and the resulting unsteady behavior of supercavitation is illustrated. It is observed that the supercavity will become unstable under the impact of the pressure wave and may collapse locally, which depends on the strength of perturbation. The huge pressure surge accompanying the collapse of supercavitation may cause the material erosion, noise, vibration and efficiency loss of operating underwater devices.
\end{abstract}

Keywords: Unsteady supercavitation; homogeneous cavitation model; compressible flow; pressure wave.

\section{Introduction}

Cavitation widely exists in a large variety of engineering applications and is an important consideration in the design of hydraulic machinery. Cavitation is usually regarded as an undesirable phenomenon. For instance, the implosion of cavitation bubble can produce huge pressure surge, which may cause severe material erosion, noise generation and other negative consequences. However, attempts also have been made to benefit from the controlled cavitation. An example is the underwater object enveloped by a supercavitation, where the skin friction drag can be reduced significantly.

Cavitation is relatively complex with rather complicated physics involved. How to construct cavitation models to accurately describe the dynamic phase change between

This is an Open Access article published by World Scientific Publishing Company. It is distributed under the terms of the Creative Commons Attribution 3.0 (CC-BY) License. Further distribution of this work is permitted, provided the original work is properly cited. 
liquid and vapour and develop robust and stable algorithms to simulate the dynamics of cavitation flow still pose challenges to both physical modelling and numerics. Cavitation has been extensively studied in the past and various cavitation models have been proposed. One popular class of models is built by adopting a phase transfer equation which reflects the phase conservation law ${ }^{1-2}$. A source term is added to the transfer equation to account for the rate of phase change between two phases, which, however, is constructed on the basis of theory of bubble dynamics. With this kind of model, the flow is treated as incompressible. This type of method has been applied to a wide range of cavitating flow computations and is found to be able to qualitatively reproduce prominent flow features observed in experiment and produce results that quantitatively agree with theoretical model prediction and experimental data. Another widely used approach is based on the single-fluid consideration with appropriate equation of state (EOS) established for both liquid flow and cavitation region. The discrepancy between the liquid compressibility effect considered in this type of model and the highly incompressible nature of real cavitation flow leads to numerical instability which needs to be treated carefully.

On the other hand, the unsteadiness of cavitation, especially that arising from an external perturbation, is far from being fully understood. In this work, a homogeneous one-fluid cavitation formulation is employed to investigate the unsteady dynamics of supercavitation subject to the introduced perturbation. It is hoped that this study can shed some light into the understanding of transient supercavitation behavior.

\section{Physical Model and Numerical Method}

\subsection{Physical model}

In this work, the cavitating flow is treated as the homogeneous mixture of two phases, liquid and vapour, which remain in kinematic and thermodynamic equilibrium. It is governed by compressible Navier-Stokes equations,

$$
\frac{\partial \mathrm{U}}{\partial t}+\frac{\partial \mathrm{F}}{\partial x}+\frac{\partial \mathrm{G}}{\partial y}+\frac{i}{y} \mathrm{~S}=\frac{\partial \mathrm{F}_{\mathrm{v}}}{\partial x}+\frac{\partial \mathrm{G}_{\mathrm{v}}}{\partial y}+\frac{i}{y} \mathrm{~S}_{\mathrm{v}}
$$

where $i$ takes value of 0 and 1 for planar and axisymmetric geometry, respectively. $\mathrm{U}$, $\mathrm{F}, \mathrm{G}$ and $\mathrm{S}$ are the vectors of conserved variables, inviscid flux in $x$ and $y$ direction, geometric source terms, respectively, and are given by,

$$
\mathrm{U}=\left(\begin{array}{c}
\rho \\
\rho u \\
\rho v
\end{array}\right) \mathrm{F}=\left(\begin{array}{c}
\rho u \\
\rho u^{2}+p \\
\rho u v
\end{array}\right) \quad \mathrm{G}=\left(\begin{array}{c}
\rho v \\
\rho u v \\
\rho v^{2}+p
\end{array}\right) \quad \mathrm{S}=\left(\begin{array}{c}
\rho v \\
\rho u v \\
\rho v^{2}
\end{array}\right) .
$$

In Eq. (2), $\rho, u, v$ and $p$ are the density, $x$ velocity component, $y$ velocity component and pressure, respectively. Energy equation is decoupled from model system 
(1) as the pressure is assumed to depend on the density only. The viscous flux vectors $\mathrm{F}_{\mathrm{v}}, \mathrm{G}_{\mathrm{v}}$ and $\mathrm{S}_{\mathrm{v}}$ are not listed to save space.

Isentropic cavitation model together with Tait EOS is used to close system (1),

$$
\rho= \begin{cases}\rho_{0}\left(\frac{p+B-A}{B}\right)^{1 / \mathrm{N}}, & p \geq p_{\text {sat }}, \\ \frac{k \rho_{\mathrm{g}}^{\mathrm{cav}}+\rho_{1}^{\mathrm{cav}}}{\left(\frac{p+B-A}{p_{\mathrm{cav}}+B-A}\right)^{-1 / \mathrm{N}}+k\left(\frac{p}{p_{\text {cav }}}\right)^{-1 / \gamma},} & p<p_{\text {sat }},\end{cases}
$$

with

$$
\frac{\alpha_{0}}{1-\alpha_{0}}=k \frac{(p+B-A)^{1 / \mathrm{N}} /\left(p_{\mathrm{cav}}+B-A\right)^{1 / \mathrm{N}}}{\left(p / p_{\mathrm{cav}}\right)^{1 / \gamma}}
$$

where $k=\alpha_{0} /\left(1-\alpha_{0}\right)$ and $\alpha_{0}$ is the known void fraction of the mixture density at $p_{\text {cav }}$. Here, $\rho_{\mathrm{g}}^{\text {cav }}$ and $\rho_{1}^{\text {cav }}$ are the associated vapour and liquid densities at the cavitation pressure $p_{\text {cav }}$, respectively. For the pure liquid phase with $p \geq p_{\text {sat }}$, Tait EOS given in Eq. (3) is used as thermodynamic closure. Cavitation or phase transition occurs as a result of local pressure drop below the saturated pressure $p_{\text {sat }}$ and is modeled by isentropic formulation presented in Eq. (3) which is proved to be mathematically sound and physically reasonable ${ }^{3}$. For liquid water, values of material-dependent parameters in Eq. (3) are set to be $N=7.15, A=10^{5} \mathrm{~Pa}, B=3.31 \times 10^{9} \mathrm{~Pa}, \rho_{0}=1000 \mathrm{Kg} / \mathrm{m}^{3}$, respectively.

The sound speed is calculated through,

$$
a=\left\{\rho\left[\frac{\alpha}{\rho_{\mathrm{sv}} \cdot a_{\mathrm{v}}^{2}}+\frac{(1-\alpha)}{\rho_{\mathrm{sw}} \cdot a_{\mathrm{w}}^{2}}\right]\right\}^{-1 / 2}
$$

where void fraction $\alpha$ is determined from mixture density definition,

$$
\rho=\alpha \rho_{\mathrm{sv}}+(1-\alpha) \rho_{\mathrm{sw}} .
$$

In Eq. (6), $\rho_{\mathrm{sw}}$ and $\rho_{\mathrm{sv}}$ are the saturated liquid and vapour densities, respectively. Viscosity coefficient for the two-phase mixture in Eq. (1) is defined as,

$$
\mu=\alpha \mu_{v}+(1-\alpha) \mu_{l}
$$

where $\mu_{v}$ and $\mu_{l}$ are viscosity coefficients of vapour and liquid, respectively.

\subsection{Numerical method}

The computational domain is discretized into a set of quadrilateral mesh, on which the system of partial differential equations (1) is integrated. Inviscid fluxes are evaluated 
using Harten-Lax-van Leer (HLL) approximate Riemann solver. Second-order monotone upstream-centered scheme for conservation laws (MUSCL) with minmod limiter is employed to reconstruct flow variables. The source terms in Eq. (1) are handled separately. Time integration is performed using two-stage Runge-Kutta algorithm and the resulting method is also second-order accurate in time.

\section{Numerical Results and Discussion}

\subsection{D axisymmetric supercavitation at freestream speed of $40 \mathrm{~m} / \mathrm{s}$}

The two-dimensional axisymmetric supercavitation around a flat-nose cylinder of radius $10 \mathrm{~mm}$ and length $150 \mathrm{~mm}$ is to be simulated with the freestream flow speed changing from $40 \mathrm{~m} / \mathrm{s}$ in the current case to $45 \mathrm{~m} / \mathrm{s}$ in the next case. The pressure and temperature of upstream flow field are $1 \mathrm{~atm}$ and $300 \mathrm{~K}$, respectively. Water enters the computational domain with the fixed velocity of $40 \mathrm{~m} / \mathrm{s}$ and exits the outlet boundary with a static pressure specified. Due to the symmetry of flow field around the cylinder axis, only the upper half of the domain is computed with non-reflecting boundary imposed on the top and symmetry boundary employed on the bottom. A quadrilateral mesh of 12,4000 cell is used in the simulation below.

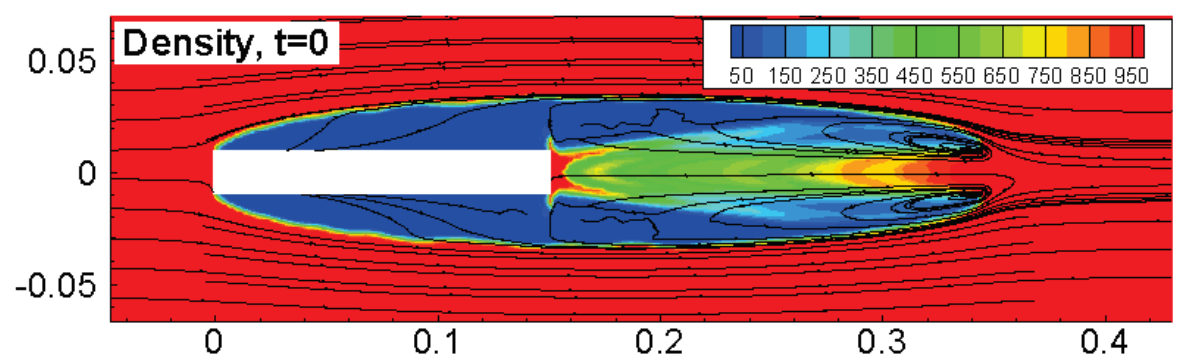

Fig. 1. An axisymmetric supercavitation enveloping a cylinder of radius $10 \mathrm{~mm}$ and length $150 \mathrm{~mm}$. The freestream speed, pressure and temperature are $40 \mathrm{~m} / \mathrm{s}$, 1 atm, and $300 \mathrm{~K}$, respectively.

The snapshot of computed density field overlaid with streamlines at $U_{\infty}=40 \mathrm{~m} / \mathrm{s}$ is illustrated in Fig. 1. The flow is characterized by a supercavitation bubble containing the whole object and a re-entrant jet in wake flow that propagates upstream but is halted by the right end of cylinder. The flow is essentially transient with captured re-entrant jet changing in shape and size slightly even after the long flow time computation. The resolved flow features show qualitative agreement with those predicted by the phase transfer equation model $^{2}$.

Next, the simulation restarts from the baseline solution depicted in Fig. 1 by increasing the freestream velocity at inlet abruptly from $40 \mathrm{~m} / \mathrm{s}$ to $45 \mathrm{~m} / \mathrm{s}$. As such, a perturbation or pressure wave is introduced, which will travels downstream and collide with the existing supercavitation bubble. The resulting unsteady evolution of supercavitating flow subject to such disturbance is demonstrated in Fig. 2 where both the density and pressure fields are depicted. At time $0.2 \mathrm{~ms}$, the pressure wave is approaching 
the cylinder head and the supercavitation does not appear to be influenced by the pressure wave with its shape largely remaining unchanged. However, the scenario at $0.4 \mathrm{~ms}$ is different. By that time, the pressure wave has swept over the whole body. The supercavity starts to collapse from its leading edge near the cylinder nose. With time moving on, the pressure wave quickly propagates downstream and finally exits the computational domain. At the same time, the cavitation bubble continues shrinking, eventually vanishing.

The pressure distribution along cylinder surface at time $0.6 \mathrm{~ms}$ is plotted in Fig. 3. It is observed that the collapse of cavitation is accompanied by a huge pressure surge with its peak magnitude being as high as 160atm, which contrasts sharply with the freestream pressure of $1 \mathrm{~atm}$. This pressure surge may cause severe material damage to the body.
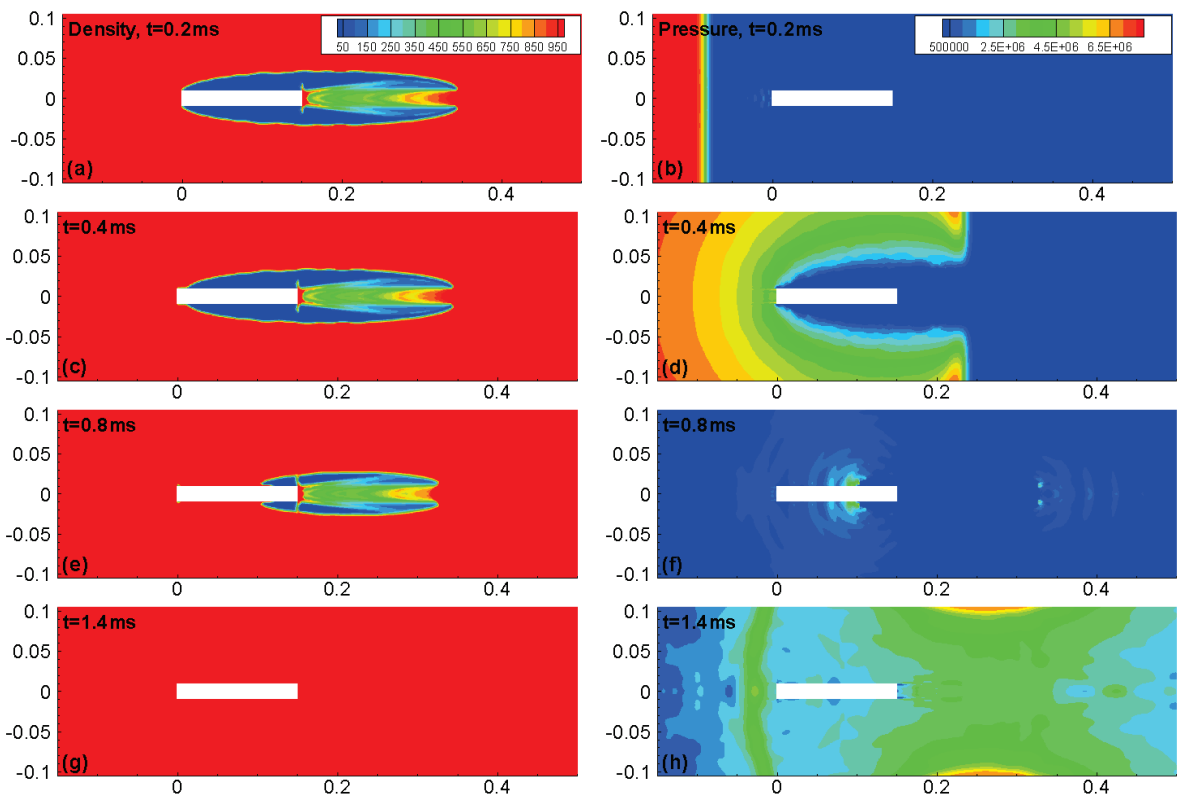

Fig. 2. The density (left column) and pressure (right column) contours for the supercavity subject to the sudden inlet velocity increase of $5 \mathrm{~m} / \mathrm{s}$.

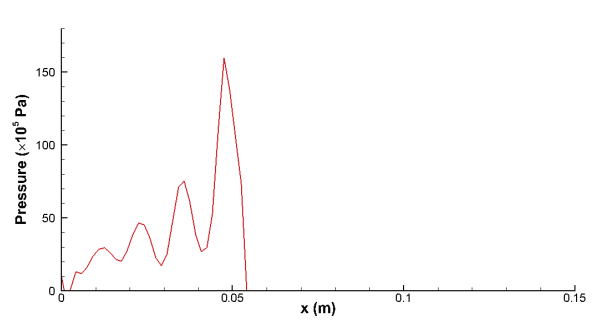

Fig. 3. The pressure distribution along the cylinder surface at time $0.6 \mathrm{~ms}$ for the case shown in Fig. 2. 


\section{2. $2 D$ axisymmetric supercavitation at freestream speed of $45 \mathrm{~m} / \mathrm{s}$}

In the second case, the flow with higher upstream speed of $45 \mathrm{~m} / \mathrm{s}$ is simulated first. Then, the inlet velocity is changed to $55 \mathrm{~m} / \mathrm{s}$, which results in a stronger pressure wave. The time sequence of predicted density field is presented in Fig. 4. The supercavity size is sensitive to the incoming flow speed and the bubble becomes longer at the higher speed. The bubble collapse process under the impact of pressure wave is similar to that in the previous case.
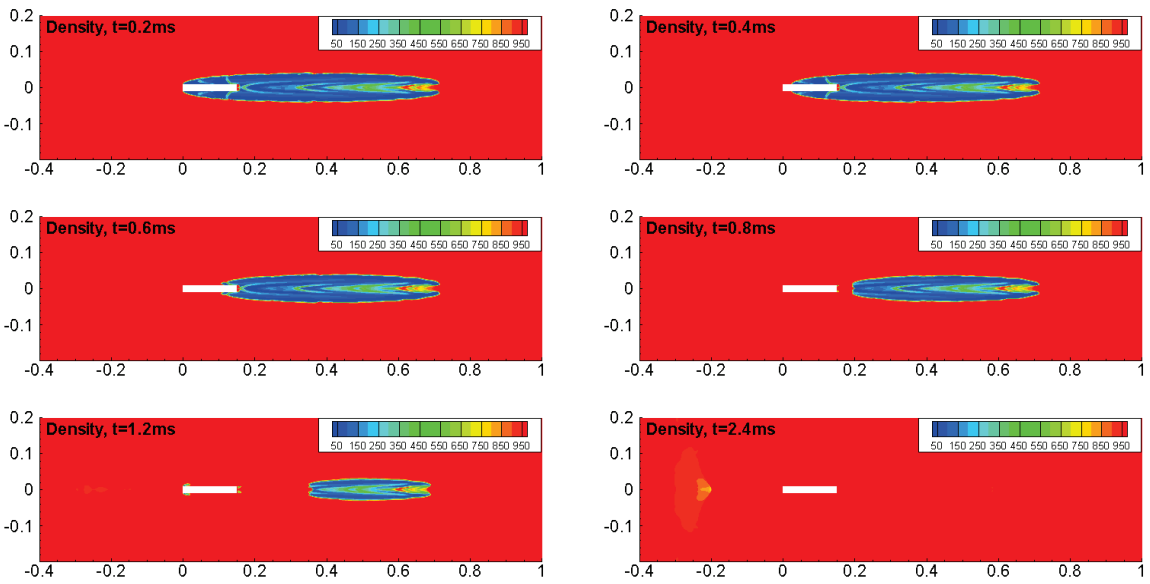

Fig. 4. The density contours showing the evolution of supercavity with the inlet velocity increased from $45 \mathrm{~m} / \mathrm{s}$ to $55 \mathrm{~m} / \mathrm{s}$.

\section{Conclusions}

The supercavitation around a flat-nose cylindrical body submerged in a water flow is simulated using the hydrodynamic code based on the homogeneous one-fluid cavitation model. The re-entrant jet behind the cylinder end is well captured. The unsteady behavior of supercavity subject to the impact of the introduced pressure wave is resolved. It is found that the local collapse of supercavitation results in extremely high pressure increase, which may cause damage to the underwater body.

\section{References}

1. R. F. Kunz, D. A. Boger and D. R. Stinebring, A preconditioned Navier-Stokes method for twophase flows with application to cavitation prediction, Comput. Fluids, 29, 849 (2000).

2. L. X. Zhang and B. C. Khoo, Computations of partial and super cavitating flows using implicit pressure-based algorithm (IPA), Comput. Fluids, 73, 1 (2013).

3. J. G. Zheng, B. C. Khoo and Z. M. Hu, Simulation of wave-flow-cavitation interaction using a compressible homogenous flow method, Commun. Comput. Phys., 14(2), 328 (2012).

4. J. Blazek, Computational fluid dynamics: principles and applications, (ELSEVIER, 2001). 